\title{
Towards the Definition of Workflows for Automation in HBIM Generation
}

\author{
Mattia Previtali $^{(\bowtie)}$ and Fabrizio Banfi \\ Department of Architecture, Built Environment and Construction Engineering, \\ Politecnico di Milano, Via Ponzio 31, 20133 Milan, Italy \\ \{mattia.previtali,fabrizio.banfi\}@polimi.it
}

\begin{abstract}
In the last years creation of as-built Building Information Modelling (BIM), and Historic Building Information Modelling (HBIM) in particular, has become a widely researched topic. In particular, the so-called "Scan.-to-BIM" procedure has received a lot of attention. This is mainly given by the fact that nowadays, terrestrial laser scanning (TLS), either static and mobile, and 3D photogrammetry are quite popular techniques to acquire building geometry raw data. However, turning a set of scans into a BIM model is still a labor-intensive and manual work. This paper presents two workflows for increasing the automation in HBIM generation. The presented approaches differ in the level of automation achieved and in the level of maturity. Indeed, while the first one presents a higher level of automation it is designed only to work in the case straight geometrical features are dominant in the scene (i.e., Manhattan world assumption holds). In addition, it is currently implemented in Matlab. On the other hand, the second one is closer to semi-automated modelling since some manual operations are still needed. However, it is implemented as a Revit Plugin and for this reason it is more user-friendly.
\end{abstract}

Keywords: HBIM $\cdot$ Automation $\cdot$ Segmentation $\cdot$ Point cloud Add-in

\section{Introduction}

In the last years creation of as-built Building Information Modelling (BIM), and Historic Building Information Modelling (HBIM) in particular, has become a widely researched topic. For historical buildings, as-found information concerning building geometry is often sparse, not accurate/updated or even missing. For this reason, the availability of a solid geometrical model is fundamental for other analysis like the definition of a conservation plan, restoration works planning, etc. In particular, the socalled "Scan.-to-BIM" procedure has received a lot of attention. This is mainly given by the fact that nowadays, terrestrial laser scanning (TLS), either static and mobile, and 3D photogrammetry are quite popular techniques to acquire raw data of a building geometry. This achievement was boosted by significant advances both in scanning technology and automated processing of images. Many issues, which were underlined in the past as critical in laser scanning, like effortful data acquisition and registration, nowadays are no more a problem due to an increase in automation of such phases. The 
reduction of instrumental cost also boosted static laser scanning in new fields. Mobile and handheld laser scanning systems (MLS) were also developed to speed up the acquisition phase using Simultaneous Localization and Mapping (SLAM) technology. Even if the accuracy of MLS is generally worst with respect to the one of TLS their higher productivity is an important advantage that allowed such platform to gain popularity. Finally, the availability of commercial software packages with a high level of automation both in the image orientation and in dense matching steps determined a new youth to photogrammetry. Even if TLS or MLS may provide reliable as-found information with a few hours (or days) of surveying operations, they are providing just raw data (point clouds) that are generally not suitable for any real application. For this purpose, a time-consuming work in needed to derive a consistent 3D model of the buildings starting from them. This procedure is still mainly manual and skilled operators are requested to turn raw point cloud into a ready-to-use BIM product. To reduce the time needed for the production of "as-found" BIM, the topic of increasing the automation of the building reconstruction pipeline starting from point clouds has been paid a lot of attention in the literature in the last years [1, 2]. However, point clouds acquired from real-world commonly feature several properties that may pose severe challenges for any algorithm designed to extract information from the raw data. The different approaches developed generally subdivide the "Scan-to-BIM" problem into four specific steps: scan preprocessing, segmentation of the point cloud, classification of detected objects and final reconstruction of the BIM model. The aim of scan preprocessing is to re-organize data to increase computational efficiency, like organizing point cloud into a $\mathrm{k}$-dimensional tree ( $\mathrm{k}-\mathrm{d}$ tree). The preprocessed point cloud is then segmented into a set of primitives (using specific criteria); next step is the classification of detected segments by using frameworks exploiting local and contextual information. Finally, labelled segments are used to combine parameters (both geometry and semantic) for the reconstruction of the BIM model using specific reconstruction algorithms. Another important bottleneck of the "Scan-to-BIM" procedure is connected to the fact that BIM modelling tools were initially designed for the design and maintenance of new constructions and not for historical building. The adaptation BIM software and tools to this latter category of buildings pose some important challenges, e.g., the lack of free-form modelling instruments in commercial BIM software packages; the low level of usability of existing tools in the case of historical constructions, etc. All those aspects boosted the research to find solutions and workaround with commercial software. In particular, several procedures and protocols were defined in order to combine modelling tools with parametric software [3, 4]. However, still lacks the definition of a systematic workflow for semi-automated and guided "Scan-to-BIM" modelling capitalizing the previously listed research. In this paper, we are presenting two workflows for increasing the automation in HBIM generation. The first one (Sect. 3) presents a higher level of automation but it is designed only to work in the case straight geometrical features are dominant (i.e., Manhattan world assumption). On the other hand, the second workflow (Sect. 4) is based on a semi-automated modelling. Main contributions presented in this paper are:

- implementation of segmentation methodology for point clouds acquired in historical buildings; 
- definition of a hierarchical semantic object classification technique based on firstorder logic sentences;

- development of a Revit Plug.in whose aim is to implement a semi-automated procedure for HBIM production.

\section{Related Work}

As previously anticipated point clouds are nowadays widely diffused as the starting point for as-built BIM reconstruction. This procedure is referred to as "Scan-to-BIM" and it is currently mainly labour-intensive and manual. For this reason, several works in literature are currently focusing on this subject $[5,6]$. Presented approaches are generally based on a multi-step architecture. The main phases are: (i) segmentation of the unstructured point cloud; (ii) semantic classification of detected elements, (iii) feature detection and clustering, (iv) creation of the model combining geometry and semantic information. In some cases the order of this phase can vary between different approaches or some steps can be performed simultaneously. This paper is mainly addressing the first two topics. Point cloud segmentation is generally defined as the process aimed at grouping similar points. The similarity between points can be related both to geometrical aspect and/or other related attributes like intensity or colour. The segmentation procedure can be designed to introduce some level of organization to data [7], or as a first step in object recognition and model fitting [8]. In the past decades, various techniques have been designed to extract surfaces from point clouds. They can be mainly grouped as [9]: (i) region growing, (ii) model-based methods, (iii) edgebased detection, (iv) and machine learning. In region growing methods $[10,11]$ it is assumed that neighbouring points presents (present similar characteristics (either geometrical or radiometric). Generally, region growing methods are a combination of two steps. Firstly, a "seed" is identified as a starting point for the following "growing" phase. Model-based methods [12, 13] assumes that man-made objects can be decomposed into geometric primitives. Starting from this, points conforming to a defined primitive are grouped as belonging to the same cluster. Identification of geometric primitives is generally performed by using robust estimators like RANSAC, MLESAC, MSAC, PROSAC, etc. As described by [14], edge-based segmentation algorithms [15] can be seen as the combination of two different steps. In the first one, the border of each surface is identified while in the second one points inside the detected boundaries are grouped into different regions. In machine learning applications, a trainer takes advantages of some examples to infer the underlying probability distribution of the characteristics of interests (features). Staring from this the trained operator can be used to take decisions for point cloud classification. Definition of features play an important role in these problems and their definition is one of the main bottlenecks Semantic classification is generally the second step of automated "Scan-toBIM" procedure. Each extracted cluster or segment derived by the first step is processed to compute its class labels (e.g., wall, floor, ceiling, etc.). Classification is generally performed by taking into consideration a set of features (e.g., geometrical, radiometric, etc.) and contextual information (e.g., neighbouring relationship) [16]. 
Classification can be performed either using heuristics or machine learning approaches. Heuristic methods [17] are taking into consideration some knowledge about the characteristics of the building objects to derive classification rules. Heuristics do not require intensive learning or large data sets in order to compute the class of an observation. However, heuristics may be biased and are often case specific. Machine learning approaches methods relies on training phase to set up a discriminant criteria among object classes. A second possibility for speeding up the modelling pipeline is the development is the development of specific add-ins clustering set of commands available in commercial software. In recent years, the diffusion of Add-ins has allowed the implementation of specific commands for modelling software such as Autodesk Revit and Mc Neel Rhinoceros with the aim to extend specific internal functionality. In particular, the development of the software's internal functions has made it possible to improve the modelling and the generation of BIM-based energy/structural analyses and the related production of schedules, databases and material computing. Most of the time, the diffusion of these additional modules for modelling applications takes place through the main web service platforms such as Autodesk App Store [18] and Food4Rhino [19]. The main apps downloaded for Autodesk Revit are mainly oriented to three categories such as (1) Reality capture/animation/rendering like, Lumion ${ }^{\circledR}$ LiveSync $\AA$ by Act-3D [20] and BIMobject by BIMobject ${ }^{\circledR}$ Corporation [21]; (2) Interoperability in which it is possible to download Import/Export contents to other software like Excel by Virtual construction and technology BIM One Inc [22], IFC 2017 and on by Autodesk, Inc [23] and 3DWarehouse-For-Revit TM by AMC Bridge; (3) Structural Simulation \& Analysis like Structural Analysis Toolkit 2018 and Advance Steel 2018 Extension and on by Autodesk, Inc [24]. On the other hand, the most downloaded add-ins in Food4Rhino are Kangaroo Physics [25], Lunchbox [26], and Grasshopper ${ }^{\circledR}$ [27]. The latter is a graphical algorithm editor tightly integrated with Rhino's 3D modelling tools and allow the creation of complex shapes using generative algorithms. Unlike RhinoScript, Grasshopper requires no knowledge of programming or scripting, but still allows designers to build form generators from the simple to the awe-inspiring. In order to automating repetitive tasks in Autodesk Revit, Dynamo add-in has been developed to create scripts that can explore complex design problems and simplify BIM work modelling flows [28]. In particular, thanks to an extremely flexible open source visual programming environment, Dynamo allows the composition of customized algorithms (procedures or formulas to solve problems) to process data and create geometries through a block graphical interface (nodes). However, based on a series of generative tests, both solutions have been discarded because they are not able to create BIM object with the direct management of laser scanning scans. For this reason, the second part of this study (development of a semiautomatic SCAN to BIM add-in) avoided the implementation of the generative process of simple/complex models through the integration of Grasshopper® and Dynamo into Autodesk Revit. The most suitable solution was the application of the grades of generation (GOG) described in a previous study applied to the generation of H-BIM [29]. The choice of the method was mainly based on three factors: (1) grades of generation GOG 9 and 10 allow better levels of automation in the generation of SCAN to BIM, (2) the grade of accuracy (GOA) reached is about $1 / 2 \mathrm{~mm}$ (deviation value between 
BIM object and point cloud), (3) Revit API libraries permit the integrations of the GOGs into Autodesk Revit's add-in and improve the modeling of complex objects.

\section{Data-Driven Automated Classification}

The data-driven approach is aimed at detecting building features (e.g., walls, roof, ceilings, etc.) from TLS data. In a first stage, planar entities are detected in the row point cloud by means of a hybrid segmentation strategy and the geometric modelling of the building is accomplished. In particular, in this step detected elements are turned into simple geometric entities like lines and polygons. In a second stage, detected elements are classified into building objects.

\subsection{Point Cloud Segmentation}

As previously anticipated one of the most important steps in an automated "Scan-toBIM" pipeline is the segmentation problem. One of the main problems connected to this task is the presence of noise and clutter in the data. For the segmentation step was can define as "noise" not only the instrumental noise but also all objects that are not of interest to the definition of the building geometry like furniture and moving people.

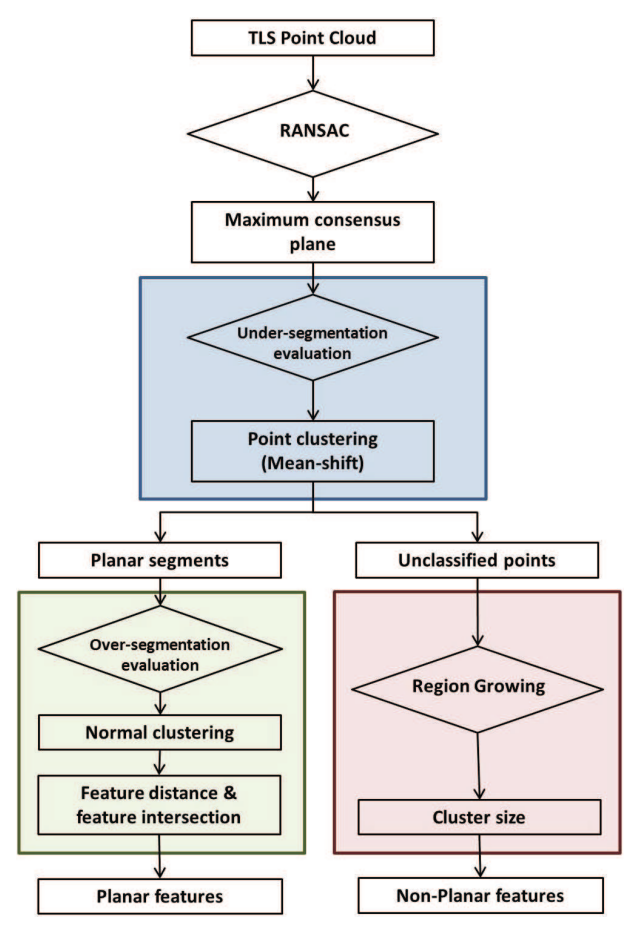

Fig. 1. Workflow of the segmentation process for historical building indoors.

The approach presented in this paper is an adaptation to historical buildings of the model-based method developed for façade segmentation that has been presented in a previous paper [30], see workflow in Fig. 1. The choice of a model based method is 
supported by a set of reasons: (i) model-based methods are less influenced by noise with respect to region growing, (ii) parameter governing the output are directly connected to geometrical characteristics of the building and (iii) in contrast to machine learning no training is requested. Traditional approaches using model-based assumption general face some bad-segmentation problem, either over-segmentation or undersegmentation. This is motivated by at least two fundamental aspects: (i) model based methods generally do not take into consideration topological information and (ii) model based algorithms only measures the consensus with respect to a geometrical model and do not take into consideration architectural priors. In order to partially cope with those problems, a modified RANSAC-based algorithm was developed to adapt model-based segmentation to face real-world problems. In particular, the developed segmentation approach is a hybrid approach that combining the robustness of RANSAC and the spatial (topological) information that is generally associated with region growing methods. Indeed, the developed approach firstly identifies the maximum consensus by using RANSAC and in a second stage classifies and groups the points by using a bottom-up clustering strategy in order to prevent under-segmentation problems. Even if an unstructured points clouds there is not a topological relationship between points we can assume that in a real-world scene points belonging to the same object should be sufficiently close to one another while points belonging to different objects should be separated by a spatial gap. For this reason, a mean shift clustering approach is adopted to cluster the different group of points. Results obtained in this way generally present a slight over-segmentation. To reduce this problem once all features are detected a grouping of the clusters is carried out to group together similar features. Object clustering is performed by evaluating three parameters: (i) similarity of normal vectors; (ii) perpendicular distance between planes; and (iii) intersection between clusters. In particular, two steps are carried out: (i) the whole group of detected segments are clustered by using the mean shift clustering algorithm using similarity of normal vectors; (ii) the perpendicular distance between features classified as different object is evaluated and if this distance is lower than the user-defined RANSAC threshold and the convex hulls of the point clusters intersects, they are recognized as a single object and merged together. In particular, the developed RANSAC strategy is used to identify planar objects (e.g. walls, floors, sidewalls, etc.) the remaining points are classified using a region growing method and grouped into objects using a strategy similar to the one previously described to cluster objects and prevent under-segmentation. Only objects whose dimension is larger than a user-defined threshold are kept as a real object while the remaining clusters are rejected and classified as "noise".

\subsection{Hierarchical Semantic Object Classification}

One of the main aspects of BIM model is their semantic characterization. For this reason, the classification and the annotation with their identity labels (e.g., 'wall') of features detected at the previous step is one of the main important points to boost the automation in the "Scan-to-BIM" pipeline. To perform such a classification a hierarchic object classification was designed starting from the consideration that each building feature has a number of attributes, which are similar within the same feature type and different with respect to other features. Similarly, also spatial relations between 
different features can be used in the classification. The main features addressed in this paper are summarized in Table 1.

Table 1. Parameters used in data processing.

\begin{tabular}{|c|c|c|c|}
\hline \multicolumn{2}{|l|}{ Features } & \multicolumn{2}{|c|}{ Spatial relationship } \\
\hline Class & Parameter(s) & Class & Parameter(s) \\
\hline Size & $\begin{array}{l}\text { - Length } \\
\text { - With } \\
\text { - Area } \\
\text { - Volume }\end{array}$ & Intersection & - Intersection of the bounding box \\
\hline Orientation & $\begin{array}{l}\text { - Normal direction of } \\
\text { the fitting plane }\end{array}$ & Angle & $\begin{array}{l}\text { - Parallelism and orthogonality } \\
\text { between normal direction of the } \\
\text { fitting plane }\end{array}$ \\
\hline Position & $\begin{array}{l}\text { - Height with respect } \\
\text { to the lowest element }\end{array}$ & Inside & - Intersection of the bounding box \\
\hline
\end{tabular}

All these information about feature attributes can be formulated in terms of firstorder logic to define the initial rules of the knowledge base classification. For example, a wall $\left(f_{1}\right)$ can be defined as a large vertical element above the floor $\left(f_{2}\right)$ and perpendicular to it:

$$
\begin{gathered}
\forall f_{1} \operatorname{IsType}(f, \text { Wall }) \\
\Rightarrow \text { IsLarge }(f) \wedge \operatorname{IsVertical}(f) \wedge \exists f_{2} \operatorname{IsAbove}\left(f_{1}, f_{2}\right) \wedge \operatorname{IsPerpendicular}\left(f_{1}, f_{2}\right) \wedge \operatorname{IsType}\left(f_{2}, \text { Floor }\right)
\end{gathered}
$$

Similarly an window 'window' $\left(f_{1}\right)$ is an intrusion of the wall $\left(f_{2}\right)$ parallel to the wall and contained into the wall itself:

$$
\begin{gathered}
\forall f_{1} \text { IsType }\left(f_{1}, \text { Window }\right) \\
\left.\Rightarrow \exists f_{2} \neg \operatorname{IsOut}\left(f_{1}, f_{2}\right) \wedge \operatorname{IsParallel}\left(f_{1}, f_{2}\right)\right) \wedge \operatorname{IsInsideO}\left(f_{1}, f_{2}\right) \wedge \operatorname{IsType}\left(f_{2}, \text { WallFacade }\right)
\end{gathered}
$$

In particular, for indoors the classification starts with evaluating both area and position of any detected objects. First, floor and ceiling are detected because both of them are horizontal (or pseudo horizontal) object. The first is characterized by lower height while the latter at the highest level. Walls are vertical objects that are in between ceiling and floor. Finally, windows and doors are detected. Indeed, while doors are classified in correspondence of gaps into walls that are connected to the floor, windows are characterized by gaps into walls that are not connected to the floor.

\subsection{Examples}

The presented segmentation and classification procedure was tested on a couple of data sets named 'Salone' and 'Volta ad ombrello'. The two datasets consist of approximately 36 million points for 'Volta ad ombrello' and approximately 42 million points 
for 'Salone'. Those data sets were acquired with a static terrestrial laser scanner namely Faro Focus 3D.

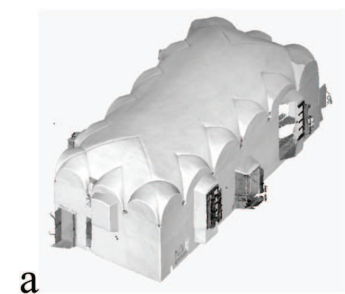

a

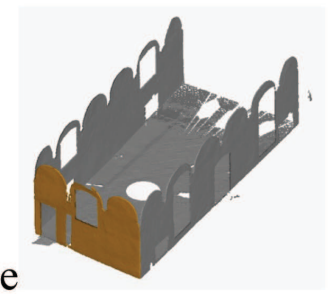

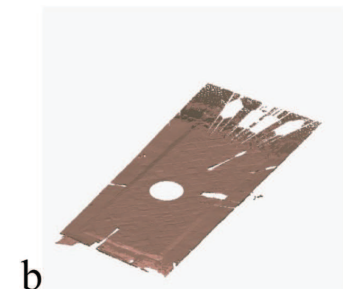

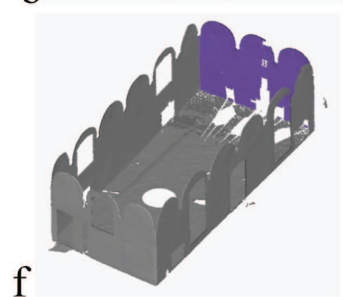

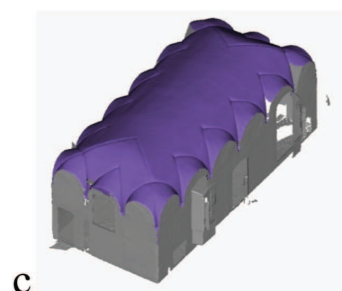

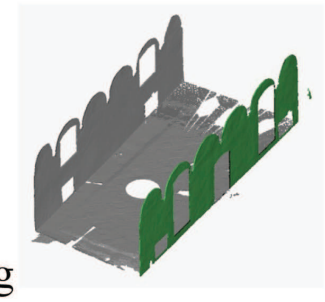

d

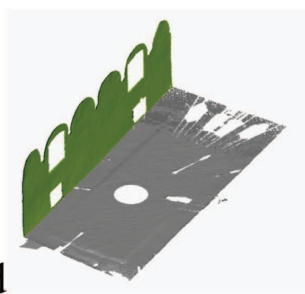

$\mathrm{h}$

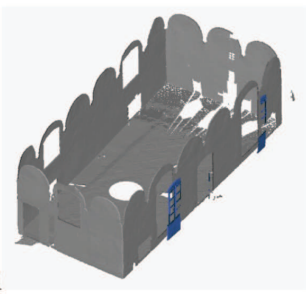

Fig. 2. 'Salone' data set. Original data (a) and results of the classification: floor (b), ceiling (c), walls $(\mathrm{d}-\mathrm{g})$ and windows $(\mathrm{h})$.

The parameters used for the segmentation are summarized in Table 2 while results are shown in Figs. 2 and 3 for the two data sets.
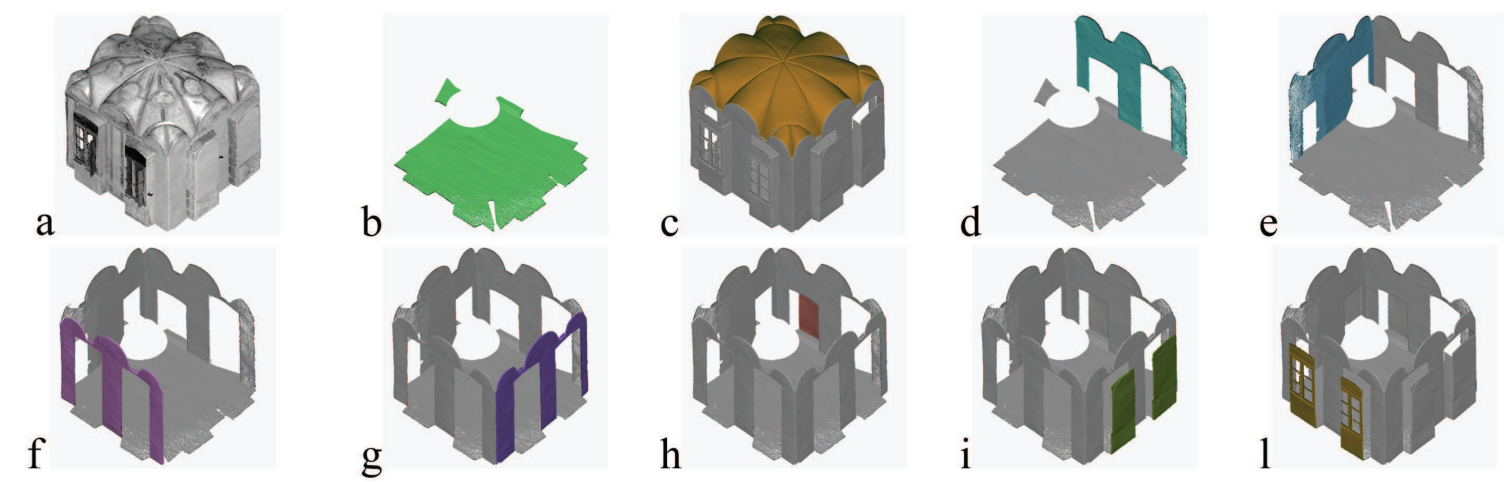

Fig. 3. 'Volta ad ombrello' data set. Original data (a) and results of the classification: floor (b), ceiling (c), walls (d-g) and doors (h-l).

Table 2. Parameters used in data processing.

\begin{tabular}{l|l}
\hline Parameter & Value \\
\hline RANSAC plane threshold $(\varepsilon)$ & $1 \mathrm{~cm}$ \\
\hline RANSAC normal threshold $(\alpha)$ & $30^{\circ}$ \\
\hline Mean shift clustering threshold $(\beta)$ & $1 \mathrm{~cm}$ \\
\hline
\end{tabular}




\section{Revit Add-in for Semi-automated Modelling}

The developed add-in structure is composed of four families able to interact with each other. Each family consists of (i) internal functions present in Revit, (ii) functions implemented by Revit APIs and finally (iii) the combined development of multiple Revit tools. Internal Revit functions, tools and functions have been recalled and hierarchized to simply the generation of as-found and as-built models. Autodesk Revit is known for the difficulty of activating modelling tools and functions. The first reason is the lack of a user-friendly interface for advanced functions. The main goal of the developed Plug-in was to achieve a more simplified activation mode of advanced tools such as automatic database generation, schedules and computing. The add-in (Fig. 4) is divided into four families.

Scan Management. It was intended to facilitate the import of point clouds and to support its setting of the BIM project in Autodesk Revit. As is known, Revit has introduced a few years ago the possibility of displaying point clouds in 3D digital space. Once the point clouds have been imported, it is not possible to apply GOG 9-10 directly. To bridge this gap, the key idea was to combine multiple functions that make point clouds more manageable. In particular, the main one was designed to help the user to the correct inserting point clouds in Revit without losing the correct georeferencing of laser scanning scans, facilitating BIM project setup operations.

Modelling (GOGs). This section integrates the GOGs, (from 1 to 10) in the software structure through the direct activation of the main BIM objects such as wall, roof, floor and ceiling. The choice to include these types of objects was dictated by their great use in SCAN to BIM projects. The other categories of objects such as a door, window, scale and supplies have not been inserted in order to manage only the $3 \mathrm{D}$ wall generation in the modelling section. The functions associated with them can be retrieved from the main interface of the software and are easily linked to the objects developed in the developed add-in. The main objective of this section was to support the user with a user-friendly guide oriented to the creation of SCAN to BIM models. In particular, for this section, it was found that the APIs for the development of modelling tools were not made available by Autodesk, thus preventing the implementation of the modelling limitations available in the Revit default interface. To bridge this gap, the writing of SCAN to BIM protocols has permitted the integration of a process based on interoperability and use of software able to generate NURBS models such as MC Neel Rhinoceros and Autodesk Autocad in the Revit's logic.

Database Generation. The main features included in this section are the automatic generation of databases and computing able to extract the numeric value such as volume, area, material and descriptive information. The main feature of a BIM is that it can extract automatic computing and information from the previously generated model. The development of the add-in has followed the SCAN to BIM process outlined in recent years thanks to the direct application of historical case studies and on existing industrial and residential buildings. 
Interoperability Levels. GOGs are based on the BIM-based import/export requirements such as the correct export of the model, to create two-dimensional drawings from the model to proper export schedules and databases in MS Access, Excell and IFC. The final objective of this section was to improve the interaction with the BIM project, external information and other types of BIM-based analysis. Mainly the new BIM utility paradigm has been supported thanks to the definition of interoperability schemes for the appropriate transfer of the SCAN to BIM model for finite element analysis and virtual reality via SAT, ACIS, DWG formats and energy analysis via IFC and GBxlm formats.

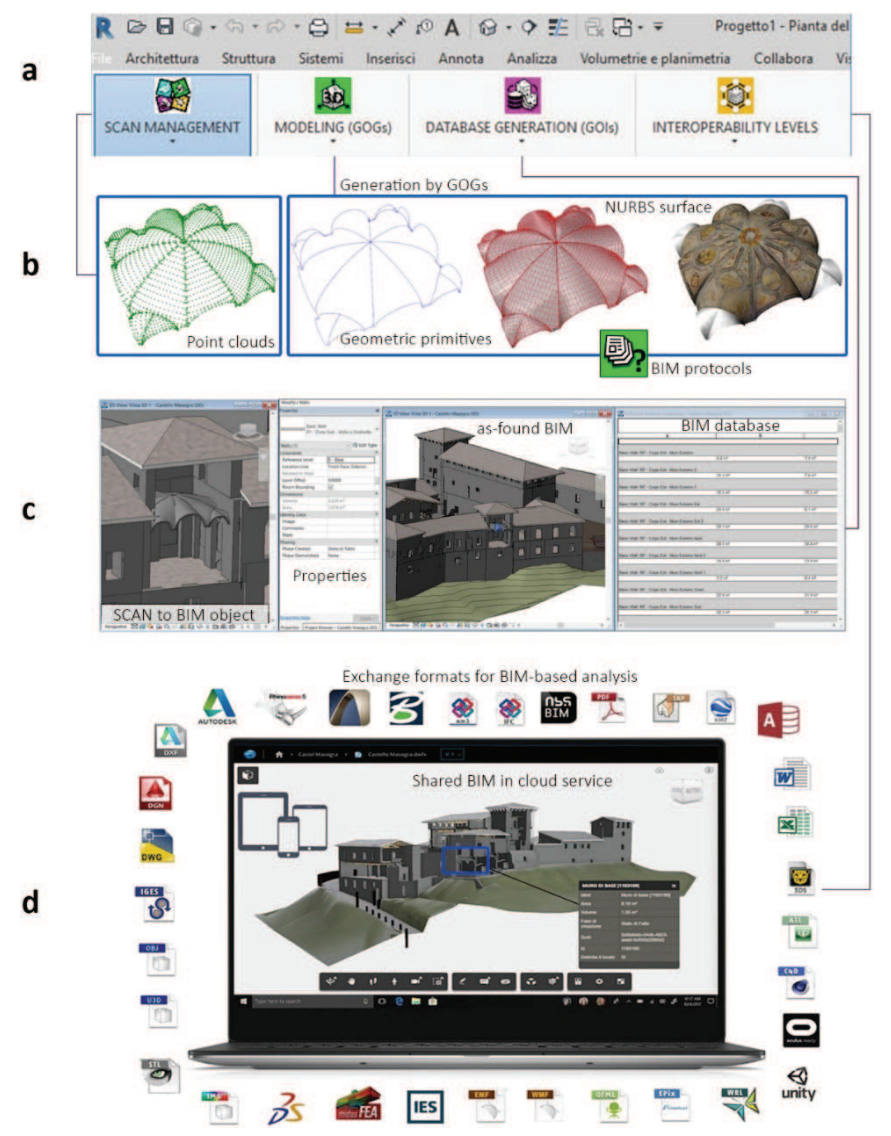

Fig. 4. The four section of the developed Revit Add-in for semi-automated modelling (a). The first section 'Scan management' (b) the second section 'Modelling GOGs' (c) implemented functions for automatic 'Database generation' and (d) Interoperability levels

\section{Conclusions}

Today, HBIM is a new paradigm in the world of built cultural heritage for the management of buildings since they are the source of geometric and semantic information. However, the generation of this models is still a labour-intensive and manual work. This research tried to contribute to increase the automation in the "Scan-to-BIM" process. In particular, two procedures were presented. The first methodology combines bottom-up modelling and top-down propagation of knowledge for segmentation and 
semantic classification of the acquired point cloud. Although the developed procedure showed a good flexibility against data quality and data density, some priors have to be associated with the top-down propagation of knowledge. In particular, it is assumed that the Manhattan world assumption holds. In the highly irregular environment the presented procedure will probably fail. Currently, the presented methodology is focusing on segmentation of the unstructured point cloud and semantic classification of detected elements. However, feature detection and clustering as well as the creation of the final model combining geometry and semantic information, which represent two crucial step in "Scan-to-BIM" process, were not tackled. This will be the subject of further works. The second methodology presented is focusing on a different approach. Indeed, it tries to speed up the BIM modelling pipeline by developing specific Add-in to commercial software packages. In this Add- in a clustering of most useful commands is performed in order to accomplish the main tasks associated to a BIM process (scan management, modelling; database generation and interoperate with other software). The developed Add-in developed a semi-automated and guided workflows for HBIM production in commercial software packages. Even if some workaround were found to reduce bottlenecks connected with commercial software some major barriers remains when facing constraints imposed by API availability.

Acknowledgements. Research leading to this results is partially funded by Regione Lombardia - Bando "Smart Living: integrazione fra produzione servizi e tecnologia nella filiera costruzionilegno-arredo-casa" approvato con d.d.u.o. n.11672 dell'15 novembre 2016 nell'ambito del progetto "HOMeBIM liveAPP: Sviluppo di una Live APP multi-utente della realtà virtuale abitativa 4D per il miglioramento di comfort-efficienza-costi, da una piattaforma cloud che controlla nel tempo il flusso BIM-sensori - ID 379270".

\section{References}

1. Volk, R., Stengel, J., Schultmann, F.: Building information modeling (BIM) for existing buildings-literature review and future needs. Autom. Constr. 38, 109-127 (2014)

2. Meschini, S., Iturralde, K., Linner, T., Bock, T.: Novel applications offered by integration of robotic tools in BIM-based design workflow for automation in construction processes. In: Advanced Construction and Building Technology for Society, p. 59 (2016)

3. Banfi, F.: Building information modelling - a novel parametric modeling approach based on 3D surveys of historic architecture. In: Ioannides, M., et al. (eds.) EuroMed 2016. LNCS, vol. 10058, pp. 116-127. Springer, Cham (2016). https://doi.org/10.1007/978-3-319-484969_10

4. Chiabrando, F., Donato, V., Lo Turco, M., Santagati, C.: Cultural heritage documentation, analysis and management using building information modelling: state of the art and perspectives. In: Ottaviano, E., Pelliccio, A., Gattulli, V. (eds.) Mechatronics for Cultural Heritage and Civil Engineering. ISCASE, vol. 92, pp. 181-202. Springer, Cham (2018). https://doi.org/10.1007/978-3-319-68646-2_8

5. Macher, H., Landes, T., Grussenmeyer, P.: From point clouds to building information models: 3D semi-automatic reconstruction of indoors of existing buildings. Appl. Sci. 7(10), 1030 (2017)

6. Bassier, M., Van Genechten, B., Vergauwen, M.: Classification of sensor independent point cloud data of building objects using random forests. J. Build. Eng. (2018) 
7. Filin, S., Pfeifer, N.: Segmentation of airborne laser scanning data using a slope adaptive neighborhood. ISPRS J. Photogramm. Remote. Sens. 60(2), 71-80 (2006)

8. Rabbani, T.: Automatic reconstruction of industrial installations using point clouds and images. Publications on Geodesy, vol. 62 (2006)

9. Grilli, E., Menna, F., Remondino, F.: A review of point clouds segmentation and classification algorithms. Int. Arch. Photogramm. Remote Sens. Spat. Inf. Sci. 42(2), W3 (2017)

10. Xiao, J., Zhang, J., Adler, B., Zhang, H., Zhang, J.: Three-dimensional point cloud plane segmentation in both structured and unstructured environments. Robot. Auton. Syst. 61(12), 1641-1652 (2013)

11. Vo, A.V., Truong-Hong, L., Laefer, D.F., Bertolotto, M.: Octree-based region growing for point cloud segmentation. ISPRS J. Photogramm. Remote Sens. 104, 88-100 (2015)

12. Chen, D., Zhang, L., Mathiopoulos, P.T., Huang, X.: A methodology for automated segmentation and reconstruction of urban 3-D buildings from ALS point clouds. IEEE J. Sel. Top. Appl. Earth Obs. Remote Sens. 7(10), 4199-4217 (2014)

13. Poux, F., Hallot, P., Neuville, R., Billen, R.: Smart point cloud: definition and remaining challenges. ISPRS Ann. Photogramm. Remote Sens. Spat. Inf. Sci. 4, 119 (2016)

14. Rabbani, T., Van Den Heuvel, F., Vosselmann, G.: Segmentation of point clouds using smoothness constraint. Int. Arch. Photogramm. Remote Sens. Spat. Inf. Sci. 36(5), 248-253 (2006)

15. Castillo, E., Liang, J., Zhao, H.: Point cloud segmentation and denoising via constrained nonlinear least squares normal estimates. In: Breuß, M., Bruckstein, A., Maragos, P. (eds.) Innovations for Shape Analysis, pp. 283-299. Springer, Heidelberg (2013). https://doi.org/ 10.1007/978-3-642-34141-0_13

16. Weinmann, M., Jutzi, B., Hinz, S., Mallet, C.: Semantic point cloud interpretation based on optimal neighborhoods, relevant features and efficient classifiers. ISPRS J. Photogramm. Remote Sens. 105, 286-304 (2015)

17. Wang, C., Cho, Y.K., Kim, C.: Automatic BIM component extraction from point clouds of existing buildings for sustainability applications. Autom. Constr. 56, 1-13 (2015)

18. Autodesk App Store Homepage. https://apps.autodesk.com/it Accessed 17 May 2018

19. Food for Rhino Homepage. http://www.food4rhino.com/. Accessed 17 May 2018

20. Lumion ${ }^{\circledR}$ LiveSync ${ }^{\circledR}$ by Act-3D download webpage in Autodesk App Store

21. BIMobject@ by BIMobject download webpage in Autodesk App Store

22. Import/Export Excel by Virtual construction and technology BIM One Inc, download webpage in Autodesk App Store

23. IFC 2018 by Autodesk, Inc. download webpage in Autodesk App Store

24. Advance Steel 2018 Extension Autodesk, Inc. download webpage, in Autodesk App Store

25. Kangaroo Physics by Daniel Piker download webpage, in Food for Rhino

26. Lunchbox by Nathan Miller download webpage, in Food for Rhino

27. Grasshopper Home page. http://www.grasshopper3d.com/. Accessed 17 May 2018

28. Dynamo Home page. http://dynamobim.org/. Accessed 17 May 2018

29. Banfi, F.: BIM orientation: grades of generation and information for different type of analysis and management process. Int. Arch. Photogramm. Remote. Sens. Spat. Inf. Sci. 42 (2/W5), 57-64 (2017)

30. Previtali, M., et al.: Automatic façade segmentation for thermal retrofit. Int. Arch. Photogramm. Remote Sens. Spat. Inf. Sci. 40, 197-204 (2013) 